\title{
Quantum walks on a programmable two-dimensional 62-qubit superconducting processor
}

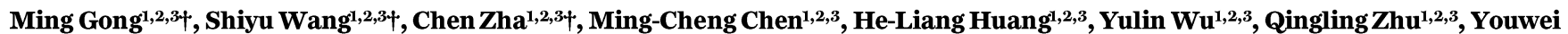
Zhao $^{1,2,3}$, Shaowei Li ${ }^{1,2,3}$, Shaojun Guo ${ }^{1,2,3}$, Haoran Qian ${ }^{1,2,3}$, Yangsen Ye ${ }^{1,2,3}$, Fusheng Chen ${ }^{1,2,3}$, Chong Ying, ${ }^{1,2,3}$, Jiale Yu ${ }^{1,2,3}$, Daojin Fan ${ }^{1,2,3}$,

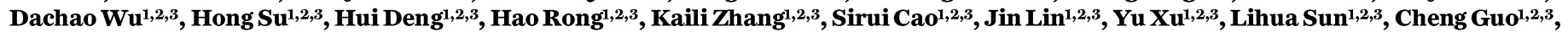
Na Li $i^{1,2,3}$, Futian Liang1,2,3, V. M. Bastidas ${ }^{4}$, Kae Nemoto ${ }^{5}$, W. J. Munro ${ }^{4,5}$, Yong-Heng Huo ${ }^{1,2,3}$, Chao-Yang Lu ${ }^{1,2,3}$, Cheng-Zhi Peng',2,3, Xiaobo $\mathrm{Zhu}^{1,2,3 *}$, Jian-Wei Pan ${ }^{1,2,3 *}$

${ }^{1}$ Hefei National Laboratory for Physical Sciences at the Microscale and Department of Modern Physics, University of Science and Technology of China, Hefei 230026, China. 2Shanghai Branch, CAS Center for Excellence in Quantum Information and Quantum Physics, University of Science and Technology of China, Shanghai 201315, China. 3Shanghai Research Center for Quantum Sciences, Shanghai 201315, China. ${ }^{4}$ NTT Basic Research Laboratories and Research Center for Theoretical Quantum Physics, 3-1 Morinosato-Wakamiya, Atsugi, Kanagawa 243-0198, Japan. ${ }^{5}$ National Institute of Informatics, 2-1-2 Hitotsubashi, Chiyoda-ku, Tokyo 101-8430, Japan.
\end{abstract}

${ }^{*}$ Corresponding authors. E-mail: xbzhu16@ustc.edu.cn (X.Z.); pan@ustc.edu.cn (J.W.P.)

†These authors contributed equally to this work.

Quantum walks are the quantum mechanical analog of classical random walks and an extremely powerful tool in quantum simulations, quantum search algorithms, and even for universal quantum computing. In our work, we have designed and fabricated an $8 \times 8$ two-dimensional square superconducting qubit array composed of 62 functional qubits. We used this device to demonstrate high fidelity single and two particle quantum walks. Furthermore, with the high programmability of the quantum processor, we implemented a Mach-Zehnder interferometer where the quantum walker coherently traverses in two paths before interfering and exiting. By tuning the disorders on the evolution paths, we observed interference fringes with single and double walkers. Our work is an essential milestone in the field, brings future larger scale quantum applications closer to realization on these noisy intermediate-scale quantum processors.

The quantum principles of superposition and entanglement allow a more powerful form of random walk, termed quantum walk (QW) (I). These walks have attracted considerable attention with many applications known in quantum transport (2), quantum simulation $(3,4)$, quantum search algorithms $(5,6)$, and even universal quantum computing ( 7 , 8 ). The universality of quantum walks has been shown with encoded quantum computation, meaning they are a universal quantum computation primitive and may give an exponential algorithmic speed-up (9). In particular, quantum walks with multiple walkers show a quantum advantage $(10,11)$ superior to ones using only a single walker. Motivated by QWs' rich potential applications, numerous proof of principle experimental demonstrations have been performed in a wide variety of hardware platforms, ranging from photonics (12, 13), trapped ions $(14,15)$, neutral atoms (16), to nuclear magnetic resonance (17), and superconducting qubits $(18,19)$.

It is well-known (20) that $\mathrm{QW}$ based quantum search algorithms require at least a two-dimensional configuration. Furthermore, easy circuit programmability with an arbitrary number of walkers is an essential requirement to encode applications, where the configuration can be changed on a walk-by-walk basis including the adjustability of tunneling amplitude and graph structure (21). Achieving both of these simultaneously have proved experimentally challenging. Superconducting circuits provide the nonlinear interaction Hamiltonian necessary for the universal quantum computation, making them one of the leading quantum computer approaches (22). Along with the real time programmability, they are now an excellent candidate system for the realization of fully configurable two-dimensional QWs.

In our work, we started with the design of a moderate scale 2D superconducting qubit array. One immediately notices the problem associated with planar wiring and how it can be realized to control all the qubits as the size of the $2 \mathrm{D}$ array increases. One solution has been 3D wiring using techniques like "flip chip" (23) or "through-silicon vias" (24). In this work, we provide an alternative technical-friendly solution based on 'pass through holes' (25). This is applied to an $8 \times 8$ qubit array (Fig. 1A) that is composed of 16 units whose circuit diagram is shown in Fig. 1B. Two of the qubits U03Q2 and U22Q1 (Fig. 1C) and one coupling resonator (between U10Q0 and U10Q3) are non-functional.

We represent the effective Hamiltonian of the qubit system using the Bose-Hubbard model as: 


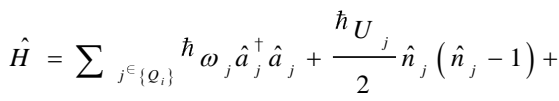

$$
\begin{aligned}
& \sum_{j^{\in} \in\left\{Q_{i}\right\}, i \in\left\{C_{Q_{i}}\right\}} \hbar_{J_{\text {eff }}^{i, j}}\left(\hat{a}_{i}^{\dagger} \hat{a}_{j}+\hat{a}_{i} \hat{a}_{j}^{\dagger}\right)
\end{aligned}
$$

where $\hat{a}_{j}^{\dagger}\left(\hat{a}_{j}\right)$ are the qubits bosonic creation (annihilation) operator, $\omega_{j}$ the $j^{\text {th }}$ qubit frequency, $U_{j}$ the anharmonicity, and $J_{\text {eff }}^{i, j}$ the effective coupling strength between $Q_{i}$ and $Q_{j}$ via a large detuned coupling resonator, where $i$ labels the qubit in group $\left\{C_{Q_{i}}\right\}$ which couples to $Q_{i}$. For the realization of continuous-time quantum walks (CTQWs), we tune all qubits to the same interaction frequency for time-independent evolution, and the effective evolution Hamiltonian is given by:

$$
\hat{H}_{\mathrm{evo}}=\sum_{i \in\left\{Q_{i}\right\}, j \in\left\{C_{Q_{i}}\right\}} \hbar_{J_{\mathrm{eff}}^{i, j}}\left(\hat{a}_{i}^{\dagger} \hat{a}_{j}+\hat{a}_{i} \hat{a}_{j}^{\dagger}\right)
$$

which forms an interference network. By setting the qubits at the interaction frequency of $5.02 \mathrm{GHz}$, we determined the effective coupling strengths $J_{\text {eff }}^{i, j}$ by measuring two-qubit swapping oscillations and established $J_{\text {eff }} / 2 \pi=2.01 \pm 0.07 \mathrm{MHz}$.

We begin by exploring continuous-time quantum walks using one and two walkers by exciting one or two qubits on U00Q0 and U33Q2. Once the initial states are prepared, we tune all qubits to the interaction frequency and allow the system to naturally evolve under Eq. (2) for a certain time. We then measure the population $\left\langle\hat{n}_{j}\right\rangle$ of all 62 qubits in their $\sigma_{z}$ basis for evolution times ranging from 0 to $600 \mathrm{~ns}$. For each time point, we performed 50,000 single-shot measurements. In Fig. 2A we present the experimental results for the twowalker QW with a comparison from numerical simulations (Fig. 2B). In the Supplementary Materials (25) we show the results for the single-walker QWs and the fidelity of the 62qubit evolution as a function of time. The high fidelity evolutions indicate the high accuracy characterization and high precision control of our system.

For quantum walks it is also extremely interesting to determine the propagation velocity of the walker (s) through the network compared to the Lieb-Robinson (LR) bound (26). Focusing on the single walker situation for simplicity, we use the two-site correlation function defined by $C_{i j}(t)=\left\langle\hat{\sigma}_{z}^{i} \hat{\sigma}_{z}^{j}\right\rangle-\left\langle\hat{\sigma}_{z}^{i}\right\rangle\left\langle\hat{\sigma}_{z}^{j}\right\rangle$ (19) to achieve this (Fig. 2C). In

Fig. 2D, we determine the propagation velocity as $22.2 \pm 2.0$ site/ us. The maximal group velocity for two-dimensional systems $(27,29)$ is given by $v_{\max }=2 \sqrt{2} J_{\text {eff }}\left(1-16 J_{\text {eff }}^{2} / 9 U^{2}\right)$ which equates to $v_{\max }=35.7 \mathrm{site} / \mu \mathrm{s}$ in our system. Now $v<v_{\max }$ clearly shows that our propagation velocity is limited by the LR bound. The difference is attributed to the short distance and disorders $(25,27,28)$.

The CTQWs demonstration establishes a solid basis for the realization of programmable QW. Furthermore, our ability to accurately vary the frequency of each qubit enables us to define propagation paths for the quantum walkers. This is critical for QW-based quantum computing where we need to deal with graph problems with different structures. In Fig. $3 \mathrm{~A}$, we define two intersecting paths in our 62-qubit superconducting processor to demonstrate a Mach-Zehnder (MZ) interferometer, where the qubits in the path are tuned to the interaction frequency of $5.02 \mathrm{GHz}$, while those not involved are biased to $4.97 \mathrm{GHz}$. After exciting the site $S$, the walker will propagate to $B S 1$ where it is split and transmitted along two spatially separated paths ( $L_{1}$ to $\left.L_{10}\right)$ and $\left(R_{1}\right.$ to $\left.R_{10}\right)$. These paths are reconnected at $B S 2$ from which the walker arrives at site $D$. The time evolution of all sites' population is measured from $t=0$ to $1000 \mathrm{~ns}$ (Fig. 3B). It clearly shows the single walker traversing both the $\{L\}$ and $\{R\}$ paths. At $t=650 \mathrm{~ns}$, a refocusing of the $\mathrm{QW}$ with the population as high as 0.43 is observed. An excellent agreement is found compared with the numerical simulations (Fig. 3D).

Our flexibility in adjusting the qubit frequencies provides another freedom we can exploit associated with the phase on paths, which is realized with the change of disorders. For the $\{R\}$ path elements we adjust the disorder of the sites $R_{1}$ to $R_{5}$ from $d_{R}$ to $5 d_{R}$ respectively, while for sites $R_{6}$ to $R_{10}$ we did the opposite changing from $5 d_{R}$ to $d_{R}$. Similar disorder changes (scaling as $d_{L}$ ) are made in the $\{L\}$ path. By controlling the disorder sizes, we measured the population on site $D$ at $t=$ $650 \mathrm{~ns}$, and observed interference fringes (Fig. 3C). To confirm the origin of these fringes, we blocked the path of $\{R\}$ on $R_{1}$ and $R_{10}$ (Fig. 3E) and found no interference fringes (Fig. $3 \mathrm{~F}$ ). Such results show that the disorder not only changes the tunneling amplitude between neighboring sites, but also provides the quantum walker a different phase accumulated in propagation that gives rise to the interference fringes. Moreover, for that interference to have occurred, our walker must have maintained coherence as it traverses a superposition of distinct spatially separated paths. The generation of those non-local correlations is essential for the development of $\mathrm{QW}$ based universal quantum computation.

The natural question that now arises is what occurs when we have multiple walkers in our MZ interferometer. We then create two walkers on sites $L_{1}$ and $R_{1}$ (Fig. 4A) by exciting these respective qubits, and then let the system evolve. We measured the population on site $D$ after $t=550 \mathrm{~ns}$, and observed the interference fringes in Fig. 4B. This is a similar pattern to what we observed in the single walker case. To determine the origin of this interference fringes in the two walker case, we performed a number of control experiments beginning with the removal of sites $B S 1$ and $S$ (Fig. $4 \mathrm{C}$ ), which stops the both walkers back propagating to take their alternate path. As shown in Fig. $4 \mathrm{D}$, no interference fringes are observed anymore. This indicates that the pattern comes from the interference between the single-particle forward 
and back propagation. Next, we created a single walker at either site $L_{1}$ or $R_{1}$ (Fig. 4, E and G) respectively, and let it walk through the interferometer. The results both clearly show interference fringes (Fig. 4, F and H). However, neither one of them nor their sum (25) are the same as what we observed in Fig. 4B. This re-enforces our observation that the two walkers present in the MZ interferometer must have interacted with each other. Such results agree well with our understanding of transmon qubit physics in the hard-core boson limit (30), where $\left|U / J_{\text {eff }}\right| \sim 120$.

To summarize, our successful demonstration of quantum walks in two dimensions and the corresponding realization of MZ interferometers clearly shows the potential of these superconducting qubit processors. With the remarkable control of not only the qubits frequencies but also the tunneling amplitude and phase between neighboring sites, these superconducting-circuit based quantum walks are elegant approach for the exploration of hard-core boson interference beyond that achievable in photonic systems. Furthermore, multi walker realizations will push us into the quantum advantage realm as the excitation number and/or the processor size increases. Finally, the demonstration of programmable quantum walks in superconducting quantum processors is an essential technological milestone providing a solid basis for more complex quantum many-body simulations, and which in the future can be further applied to quantum search algorithms and even universal quantum computing.

\section{REFERENCES AND NOTES}

1. Y. Aharonov, L. Davidovich, N. Zagury, Quantum random walks. Phys. Rev. A 48, 1687-1690 (1993). doi:10.1103/PhysRevA.48.1687 Medline

2. O. Mülken, A. Blumen, Continuous-time quantum walks: Models for coherent transport on complex networks. Phys. Rep. 502, 37-87 (2011). doi:10.1016/i.physrep.2011.01.002

3. A. Aspuru-Guzik, P. Walther, Photonic quantum simulators. Nat. Phys. 8, 285-291 (2012). doi:10.1038/nphys2253

4. N. Lambert, Y.-N. Chen, Y.-C. Cheng, C.-M. Li, G.-Y. Chen, F. Nori, Quantum biology. Nat. Phys. 9, 10-18 (2013). doi:10.1038/nphys2474

5. N. Shenvi, J. Kempe, K. B. Whaley, Quantum random-walk search algorithm. Phys. Rev. A 67, 052307 (2003). doi:10.1103/PhysRevA.67.052307

6. A. M. Childs, J. Goldstone, Spatial search by quantum walk. Phys. Rev. A 70, 022314 (2004). doi:10.1103/PhysRevA.70.022314

7. A. M. Childs, Universal computation by quantum walk. Phys. Rev. Lett. 102, 180501 (2009). doi:10.1103/PhysRevLett.102.180501 Medline

8. A. M. Childs, D. Gosset, Z. Webb, Universal computation by multiparticle quantum walk. Science 339, 791-794 (2013). doi:10.1126/science.1229957 Medline

9. A. M. Childs et al., in Proceedings of the thirty-fifth annual ACM symposium on Theory of computing (2003).

10. P. P. Rohde, T. C. Ralph, Error tolerance of the boson-sampling model for linear optics quantum computing. Phys. Rev. A 85, 022332 (2012). doi:10.1103/PhysRevA.85.022332

11. H.-S. Zhong, H. Wang, Y. H. Deng, M. C. Chen, L. C. Peng, Y. H. Luo, J. Qin, D. Wu, X. Ding, Y. Hu, P. Hu, X. Y. Yang, W. J. Zhang, H. Li, Y. Li, X. Jiang, L. Gan, G. Yang, L. You, Z. Wang, L. Li, N. L. Liu, C. Y. Lu, J. W. Pan, Quantum computational advantage using photons. Science 370, 1460-1463 (2020). Medline

12. A. Peruzzo, M. Lobino, J. C. F. Matthews, N. Matsuda, A. Politi, K. Poulios, X.-Q. Zhou, Y. Lahini, N. Ismail, K. Wörhoff, Y. Bromberg, Y. Silberberg, M. G. Thompson,
J. L. OBrien, Quantum walks of correlated photons. Science 329, 1500-1503 (2010). doi:10.1126/science.1193515 Medline

13. C. Chen, X. Ding, J. Qin, Y. He, Y.-H. Luo, M.-C. Chen, C. Liu, X.-L. Wang, W.-J. Zhang, H. Li, L.-X. You, Z. Wang, D.-W. Wang, B. C. Sanders, C.-Y. Lu, J.-W. Pan, Observation of Topologically Protected Edge States in a Photonic TwoDimensional Quantum Walk. Phys. Rev. Lett. 121, 100502 (2018). doi:10.1103/PhysRevLett.121.100502 Medline

14. H. Schmitz, R. Matjeschk, Ch. Schneider, J. Glueckert, M. Enderlein, T. Huber, T. Schaetz, Quantum walk of a trapped ion in phase space. Phys. Rev. Lett. 103, 090504 (2009). doi:10.1103/PhysRevLett.103.090504 Medline

15. F. Zähringer, G. Kirchmair, R. Gerritsma, E. Solano, R. Blatt, C. F. Roos, Realization of a quantum walk with one and two trapped ions. Phys. Rev. Lett. 104, 100503 (2010). doi:10.1103/PhysRevLett.104.100503 Medline

16. M. Karski, L. Förster, J.-M. Choi, A. Steffen, W. Alt, D. Meschede, A. Widera, Quantum walk in position space with single optically trapped atoms. Science 325 , 174-177 (2009). doi:10.1126/science.1174436 Medline

17. C. A. Ryan, M. Laforest, J.-C. Boileau, R. Laflamme, Experimental implementation of a discrete-time quantum random walk on an NMR quantum-information processor. Phys. Rev. A 72, 062317 (2005). doi:10.1103/PhysRevA.72.062317

18. V. V. Ramasesh, E. Flurin, M. Rudner, I. Siddiqi, N. Y. Yao, Direct Probe of Topological Invariants Using Bloch Oscillating Quantum Walks. Phys. Rev. Lett. 118, 130501 (2017). doi:10.1103/PhysRevLett.118.130501 Medline

19. Z. Yan, Y.-R. Zhang, M. Gong, Y. Wu, Y. Zheng, S. Li, C. Wang, F. Liang, J. Lin, Y. Xu, C. Guo, L. Sun, C.-Z. Peng, K. Xia, H. Deng, H. Rong, J. Q. You, F. Nori, H. Fan, X. Zhu, J.-W. Pan, Strongly correlated quantum walks with a 12-qubit superconducting processor. Science 364, 753-756 (2019). doi:10.1126/science.aaw1611 Medline

20. A. Tulsi, Faster quantum-walk algorithm for the two-dimensional spatial search. Phys. Rev. A 78, 012310 (2008). doi:10.1103/PhysRevA.78.012310

21. M. S. Underwood, D. L. Feder, Bose-Hubbard model for universal quantum-walkbased computation. Phys. Rev. A 85, 052314 (2012) doi:10.1103/PhysRevA.85.052314

22. F. Arute, K. Arya, R. Babbush, D. Bacon, J. C. Bardin, R. Barends, R. Biswas, S. Boixo, F. G. S. L. Brandao, D. A. Buell, B. Burkett, Y. Chen, Z. Chen, B. Chiaro, R. Collins, W. Courtney, A. Dunsworth, E. Farhi, B. Foxen, A. Fowler, C. Gidney, M. Giustina, R. Graff, K. Guerin, S. Habegger, M. P. Harrigan, M. J. Hartmann, A. Ho, M. Hoffmann, T. Huang, T. S. Humble, S. V. Isakov, E. Jeffrey, Z. Jiang, D. Kafri, K. Kechedzhi, J. Kelly, P. V. Klimov, S. Knysh, A. Korotkov, F. Kostritsa, D. Landhuis, M. Lindmark, E. Lucero, D. Lyakh, S. Mandrà, J. R. McClean, M. McEwen, A. Megrant, X. Mi, K. Michielsen, M. Mohseni, J. Mutus, O. Naaman, M. Neeley, C. Neill, M. Y. Niu, E. Ostby, A. Petukhov, J. C. Platt, C. Quintana, E. G. Rieffel, P. Roushan, N. C. Rubin, D. Sank, K. J. Satzinger, V. Smelyanskiy, K. J. Sung, M. D. Trevithick, A. Vainsencher, B. Villalonga, T. White, Z. J. Yao, P. Yeh, A. Zalcman, H. Neven, J. M. Martinis, Quantum supremacy using a programmable superconducting processor. Nature 574, 505-510 (2019). doi:10.1038/s41586019-1666-5 Medline

23. B. Foxen et al., Quantum. Sci. Tech. (Paris) 3, 014005 (2017).

24. M. Vahidpouretal., arXiv:1708.02226 (2017).

25. Materials and methods are available as supplementary materials.

26. E. H. Lieb, D. W. Robinson, The finite group velocity of quantum spin systems. Commun. Math. Phys. 28, 251-257 (1972). doi:10.1007/BF01645779

27. M. Cheneau, P. Barmettler, D. Poletti, M. Endres, P. Schauss, T. Fukuhara, C. Gross, I. Bloch, C. Kollath, S. Kuhr, Light-cone-like spreading of correlations in a quantum many-body system. Nature 481, 484-487 (2012). doi:10.1038/nature10748 Medline

28. C. K. Burrell, J. Eisert, T. J. Osborne, Information propagation through quantum chains with fluctuating disorder. Phys. Rev. A 80, 052319 (2009). doi:10.1103/PhysRevA.80.052319

29. Y. Takasu, T. Yagami, H. Asaka, Y. Fukushima, K. Nagao, S. Goto, I. Danshita, Y Takahashi, Energy redistribution and spatiotemporal evolution of correlations after a sudden quench of the Bose-Hubbard model. Sci. Adv. 6, eaba9255 (2020). doi:10.1126/sciadv .aba9255 Medline

30. Y. Lahini, M. Verbin, S. D. Huber, Y. Bromberg, R. Pugatch, Y. Silberberg, Quantum walk of two interacting bosons. Phys. Rev. A 86, 011603 (2012) doi:10.1103/PhysRevA.86.011603 


\section{ACKNOWLEDGMENTS}

The authors thank the USTC Center for Micro- and Nanoscale Research and Fabrication for supporting the sample fabrication. The authors also thank QuantumCTek Co., Ltd., for supporting the fabrication and the maintenance of room-temperature electronics. Funding: This research was supported by the National Key R\&D Program of China (Grant No. 2017YFA0304300), the Chinese Academy of Sciences, Anhui Initiative in Quantum Information Technologies,

Technology Committee of Shanghai Municipality, National Science Foundation of China (Grants No. 11905217, No. 11774326), Shanghai Municipal Science and

Technology Major Project (Grant No. 2019SHZDZX01), Natural Science

Foundation of Shanghai (Grant No. 19ZR1462700), and Key-Area Research and Development Program of Guangdong Provice (Grant No. 2020B0303030001).

This work was also supported in part by the Japanese MEXT Quantum Leap Flagship Program (MEXT Q-LEAP), Grant No. JPMXS0118069605. Author contributions: X.Z. and J.-W.P. conceived the research. M.G., S.W, C.Z., M.-C.C., and X.Z. designed the experiment. S.W., M.G., Q.Z., Y.Z., Y.Y., F.C., C.Y., and X.Z. designed the sample. S.W., C.Z., H.R., H.D., K.Z., S.C., and Y.-H.H. prepared the sample. S.G., H.Q., and H.D. prepared the Josephson parametric amplifiers. Y.W. developed the programming platform for the experiments. M.G., S.W., C.Z., Y.Z., S.L., C.Y., J.Y., D.F., D.W., and H.S. contributed to the building of the ultra lowtemperature and low-noise measurement system. J.L., Y.X., F.L., C.G., L.S., N.L., and C.-Z.P. developed the room-temperature electronics. M.G., S.W., C.Z., M.-

C.C., H.-L.H., V.M.B., K.N., W.M., C.-Y.L., and X.Z. performed the data analysis. All authors contributed to the discussions of the results and the preparation of the manuscripts. X.Z. and J.-W.P. supervised the whole project. Competing interests: None declared. Data and materials availability: All data needed to evaluate the conclusions in the paper are present in the paper or the supplementary materials.

\section{SUPPLEMENTARY MATERIALS}

science.sciencemag.org/cgi/content/full/science.abg7812/DC1

Materials and Methods

Supplementary Text

Figs. S1 to S13

Table S1

References

Movie S1 to S4

28 January 2021; accepted 20 April 2021

Published online 6 May 2021

$10.1126 /$ science.abg7812 


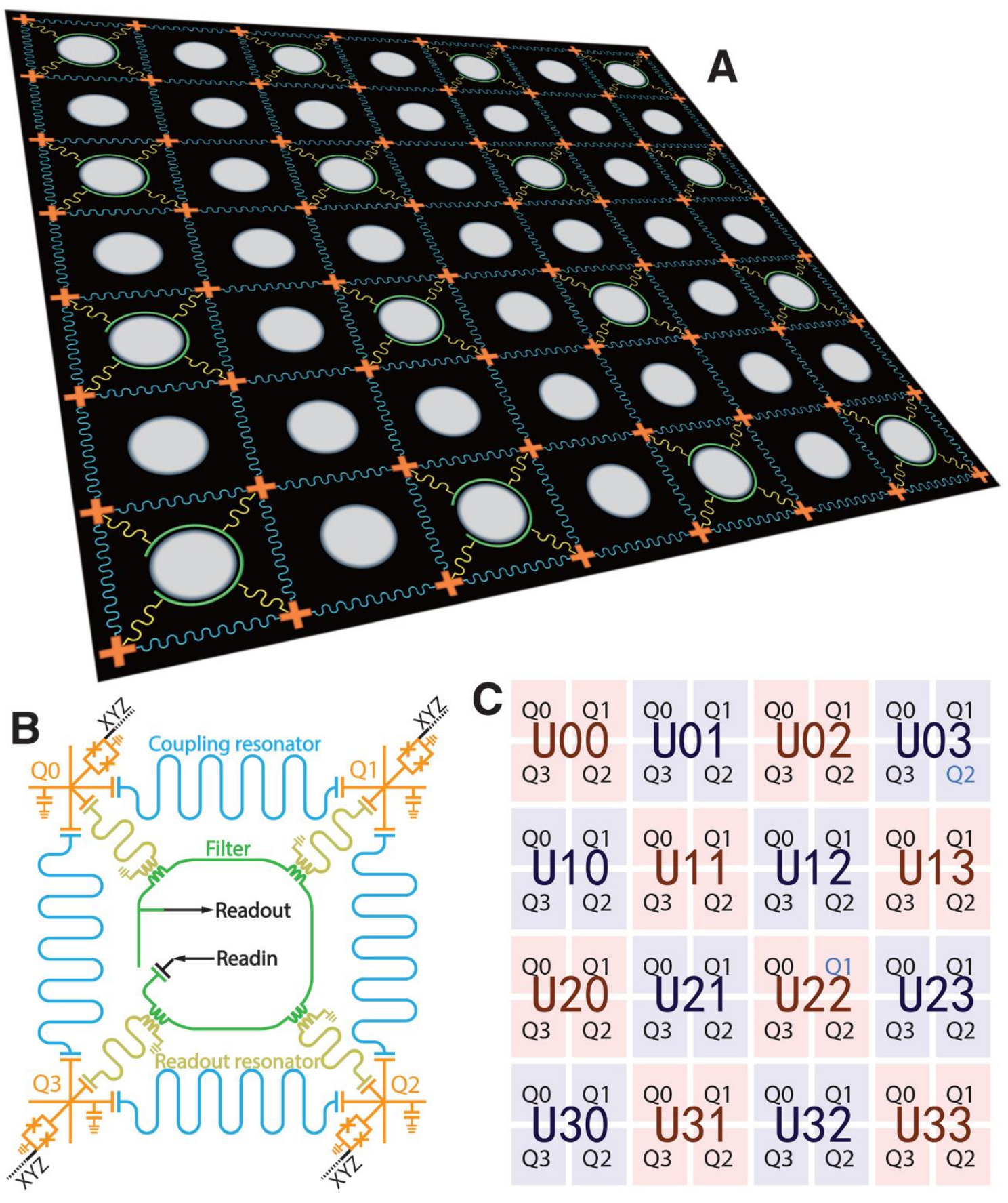

Fig. 1. The layout and architecture of the superconducting quantum processor. (A) The schematic diagram of the 2D superconducting quantum processor. The orange crosses represent the qubits arranged in an $8 \times 8$ array. The gray circles are the pass through holes (25) for 3D wiring. The electrodes for wiring are not shown for simplification. (B) The circuit diagram of a unit of the qubit array. Each qubit (orange) has an $X Y Z$ control line (black) for microwave and pulse control. The qubit couples to an individual $\lambda / 4$ readout resonator (yellow) which in turn are commonly coupled to a filter (green). Two neighboring qubits are dispersively coupled to each other through a $\lambda / 2$ coupling resonator (blue). (C) The labels of qubits. Two broken qubits, namely $\cup 03 \mathrm{Q} 2$ and U22Q1, are marked in blue. 

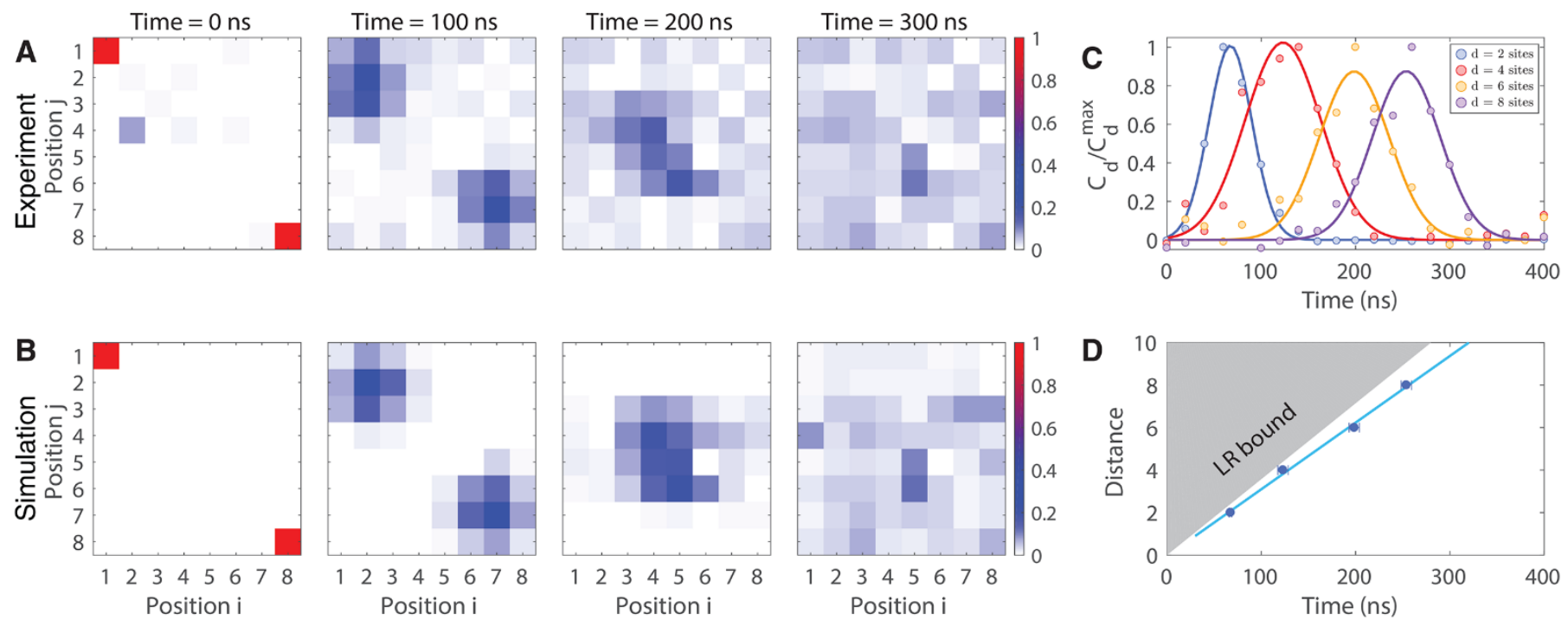

$\begin{array}{llllllll}1 & 2 & 3 & 5 & 6 & 7 & 8\end{array}$ Position $\mathrm{i}$
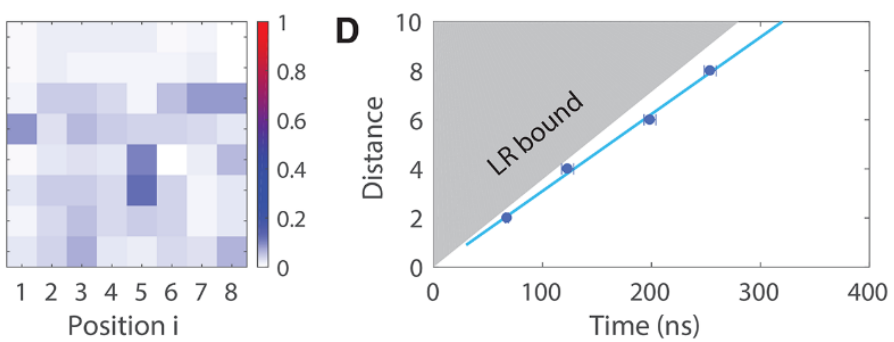

Fig. 2. Quantum walks on a 2D superconducting qubits array. (A) The evolution of the measured populations $\left\langle n_{j}\right\rangle$ of all qubits at times $t=0 \mathrm{~ns}, 100 \mathrm{~ns}, 200 \mathrm{~ns}$, and $300 \mathrm{~ns}$, respectively, with the two walkers initialized on qubits U00Q0 and U33Q2. (B) Numerical simulation of the qubits population evolution under the same conditions as (A). (C) The correlation function as a function of time in the case of single-particle QW. The blue, red, orange, and purple circles represent the measured data points for the correlation function between the initial excitation site and the sites on the diagonal with distance $d=\sqrt{2}, 2 \sqrt{2}, 3 \sqrt{2}, 4 \sqrt{2}$ sites, respectively. The corresponding solid curves are Gaussian fittings to the data, and the propagation fronts are the center of the Gaussian fittings. (D) The propagation velocity and the Lieb-Robinson bounds. Using a linear fit (blue line) of the propagation fronts (blue circles) with distance, we determine the propagation velocity as $22.2 \pm 2.0$ site/ $\mu$ s. The gray shadow shows Lieb-Robinson bound with $v_{\max }=35.7$ site $/ \mu \mathrm{s}$. 

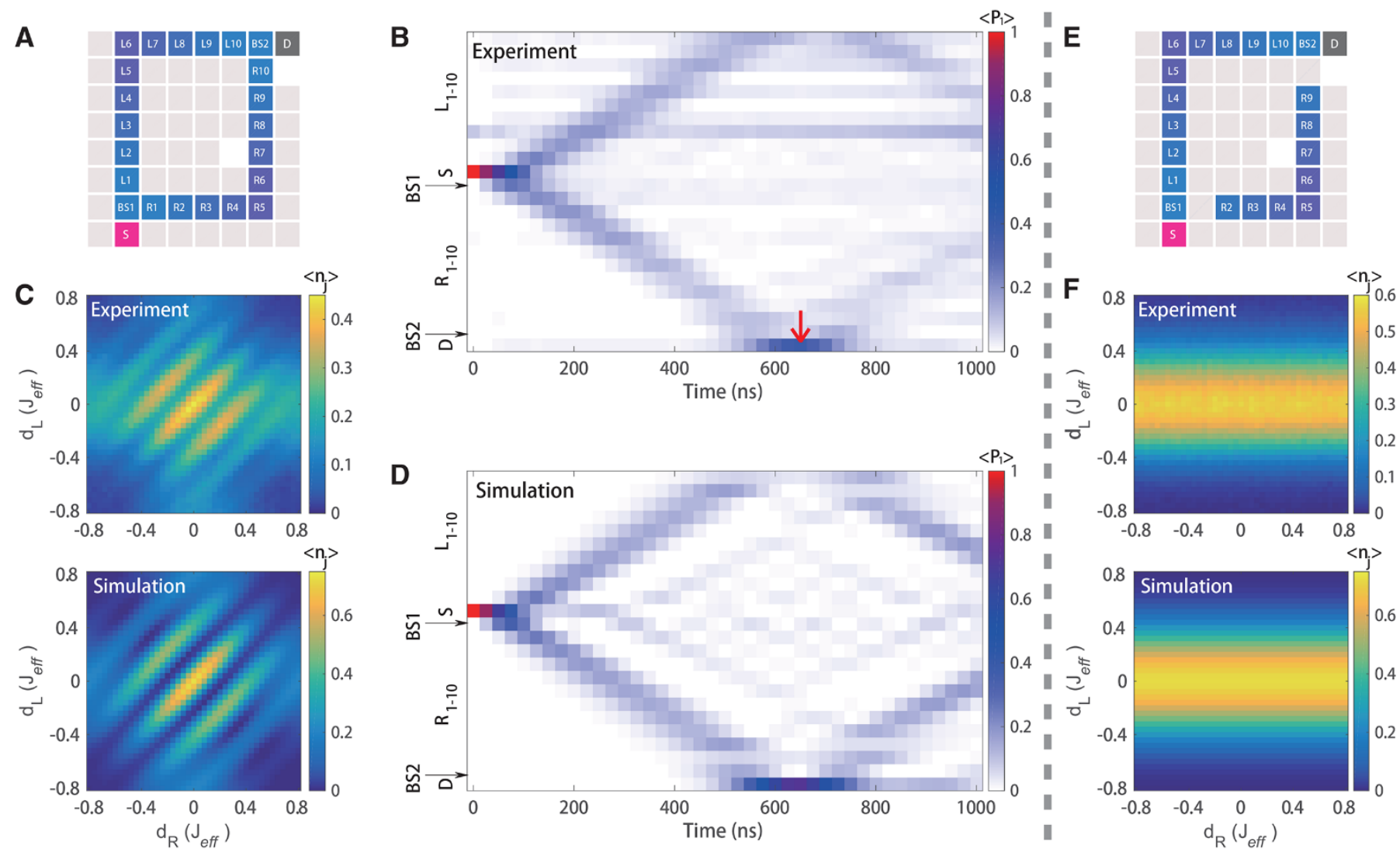

Fig. 3. Single-particle Mach-Zehnder interferometer. (A) The circuit diagram of the programmable paths for the realization of the single-particle Mach-Zehnder interferometer in a qubit array. In (B) and (D) we illustrate the dynamics evolution of population $\left\langle n_{j}\right\rangle$ of all relevant sites in experiment and simulation, respectively. The red arrow marks the time $t=650$ ns when the population of $D$ is maximized. (C) The experimental and simulated population of $D$ at $t=650$ ns under different disorder steps in two paths. (E) The circuit diagram of the interferometer with the $\{R\}$ path blocked at $R_{1}$ and $R_{10}$. (F) The experimental and simulated population of $D$ under different disorder steps in two paths with $\{R\}$ path blocked. 
A

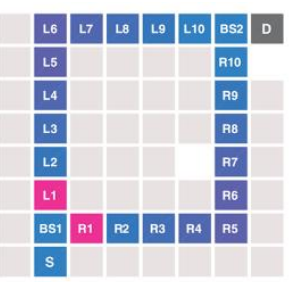

C

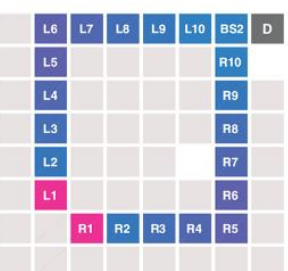

E

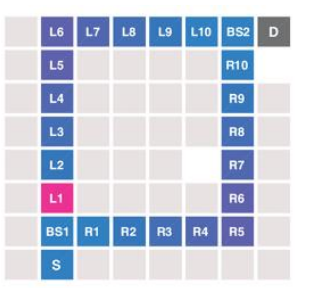

G
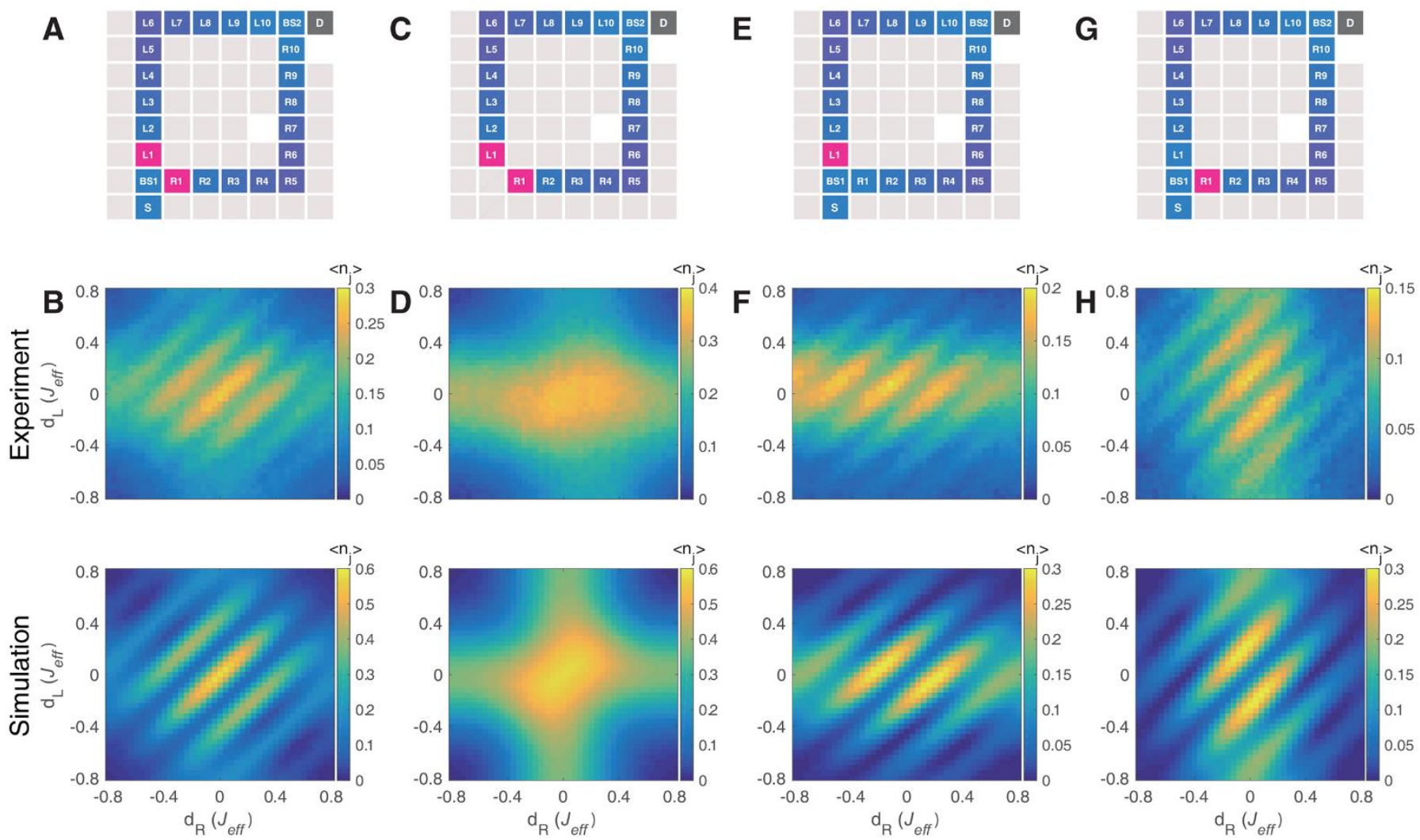

Fig. 4. Two walkers in the Mach-Zehnder interferometer. (A, C, E, and G) Circuit diagrams of the programmable paths for the Mach-Zehnder interferometer. The initial state composed of either a single or double walker is prepared by the excitation of the sites marked with pink. (B, D, F, and $\mathrm{H}$ ) The experimental and numerically simulated population of $D$ at $t=550$ ns are shown for the various configurations below the circuit diagram. 